\title{
Estudo de estabilidade acelerada de solução contendo extrato de plantas da Medicina
}

\section{Tradicional Chinesa}

\author{
Accelerated stability study of a solution containing plant extract from Traditional Chinese
}

\section{Medicine}

Estudio acelerado de estabilidade de solucíon que contiene extracto de planta de Medicina

\section{Tradicional China}

Recebido: 10/10/2021 | Revisado: 17/10/2021 | Aceito: 21/10/2021 | Publicado: 24/10/2021

\author{
Bianca Ishida Machado \\ ORCID: https://orcid.org/0000-0002-4358-796X \\ Centro Universitário Ingá, Brasil \\ E-mail: biancamachado82001@ gmail.com \\ Clara Beatriz de Lima \\ ORCID: https://orcid.org/0000-0003-0566-0799 \\ Universidade Estadual de Maringá, Brasil \\ E-mail:limacb21@gmail.com \\ Ana Carolina Guidi \\ ORCID: https://orcid.org/0000-0003-4264-9599 \\ Universidade Estadual de Maringá, Brasil \\ E-mail: carol_guidi@hotmail.com \\ Ana Paula Margioto Teston \\ ORCID: https://orcid.org/0000-0003-0212-2362 \\ Centro Universitário Ingá, Brasil \\ E-mail: farmacia@uninga.edu.br \\ Francine Maery Dias Ferreira-Romanichen \\ ORCID: https://orcid.org/0000-0001-6150-1982 \\ Centro Universitário Ingá, Brasil \\ E-mail: prof.francineferreira@ uninga.edu.br \\ João Carlos Palazzo de Mello \\ ORCID: https://orcid.org/0000-0002-7532-3395 \\ Universidade Estadual de Maringá, Brasil \\ E-mail: mello@uem.br \\ Daniela Cristina de Medeiros Araújo \\ ORCID: https://orcid.org/0000-0001-8602-035X \\ Centro Universitário Ingá, Brasil \\ E-mail: danielamedeiros@ hotmail.com
}

\begin{abstract}
Resumo
A Medicina Tradicional Chinesa (MTC) é uma ciência que passou seus milhares de anos de existência agregando conhecimentos de suas práticas, que se baseiam no princípio do equilíbrio das energias yin e yang. A estabilidade é o fator que determina a compatibilidade entre os princípios ativos com seus excipientes e embalagens, quando não há essa compatibilidade, a conservação de uma formulação é diretamente afetada. Sendo assim, o objetivo deste trabalho foi realizar um estudo de estabilidade acelerado ( 0 a 6 meses; $40{ }^{\circ} \mathrm{C}, 25 \%$ UR) de solução contendo extrato de seis plantas da MTC, por meio de testes de controle de qualidade físico-químicos (características organolépticas, $\mathrm{pH}$, densidade e viscosidade) e microbiológicos (para fungos e bactérias), seguindo as metodologias descritas na Farmacopeia Brasileira $6^{\mathbf{a}}$ edição. Foi realizada também a análise do perfil cromatográfico por CLAE. Os resultados observados nas avaliações físico-químicas realizadas durante o período de estudo (6 meses) demonstraram estabilidade adequada nos parâmetros organolépticos, $\mathrm{pH}$ e densidade. A viscosidade observada foi diferente de outros trabalhos, mas é aceitável para soluções e manteve-se estável durante os tempos analisados. Já na avaliação por cromatografia, observou-se indícios de degradação dos ativos após o tempo 1 mês, não apresentando estabilidade diante das condições de temperatura e umidade utilizadas durante o estudo. Na avaliação da qualidade microbiológica, não foi constatado o crescimento de fungos ou bactérias nos tempos analisados.
\end{abstract}

Palavras-chave: Fitoterápico; Cromatografia líquida de alta eficiência (CLAE); Controle de qualidade. 


\begin{abstract}
Traditional Chinese Medicine (TCM) is a science that has spent its thousands of years of existence aggregating knowledge of its practices, which are based on the principle of balancing yin and yang energies. Stability is the factor that determines the compatibility between the active principles with their excipients and packaging, when there is no such compatibility, the preservation of a formulation is directly affected. Therefore, the objective of this work was to carry out an accelerated stability study ( 0 to 6 months; $40{ }^{\circ} \mathrm{C}, 25 \%$ UR) of a solution containing extract of six MTC plants, through physical-chemical (organoleptic characteristics, $\mathrm{pH}$, density and viscosity) and microbiological (for fungi and bacteria) quality control tests, following the methodologies described in the Brazilian Pharmacopoeia 6th edition. The analysis of the chromatographic profile by HPLC was also performed. The results observed in the physicochemical evaluations carried out during the study period (6 months) demonstrated adequate stability in organoleptic parameters, $\mathrm{pH}$ and density. The observed viscosity was different from other works, but it is acceptable for solutions and remained stable during the analyzed times. In the evaluation by chromatography, there was evidence of degradation of the assets after time 1 month, not showing stability under the conditions of temperature and humidity used during the study. In the evaluation of microbiological quality, the growth of fungi or bacteria was not observed in the analyzed times.
\end{abstract}

Keywords: Herbal medicine; High performance liquid chromatography (HPLC); Quality control.

\title{
Resumen
}

La Medicina Tradicional China (MTC) es una ciencia que ha pasado sus miles de años de existencia agregando el conocimiento de sus prácticas, que se basan en el principio de equilibrar las energías yin y yang. La estabilidad es el factor que determina la compatibilidad entre los principios activos con sus excipientes y el envase, cuando no existe tal compatibilidad, la conservación de una formulación se ve directamente afectada. Por tanto, el objetivo de este trabajo fue realizar un estudio de estabilidad acelerada ( 0 a 6 meses; $40{ }^{\circ} \mathrm{C}, 25 \%$ UR) de una solución que contiene extracto de seis plantas de MTC, mediante pruebas físico-químicas (características organolépticas, $\mathrm{pH}$, densidad y viscosidad) y de control de calidad microbiológico, siguiendo las metodologías descritas en la $6^{\mathrm{a}}$ edición de la Farmacopea Brasileña. También se realizó el análisis del perfil cromatográfico por HPLC. Los resultados observados en las evaluaciones fisicoquímicas realizadas durante el período de estudio (6 meses) demostraron una adecuada estabilidad en los parámetros organolépticos, $\mathrm{pH}$ y densidad. La viscosidad observada fue diferente a otros trabajos, pero es aceptable para soluciones y se mantuvo estable durante los tiempos analizados. En la evaluación por cromatografía, se evidenció degradación de los activos después del tiempo 1 mes, no mostrando estabilidad en las condiciones de temperatura y humedad utilizadas durante el estudio. En la evaluación de la calidad microbiológica, no se observó crecimiento de hongos o bacterias en los tiempos analizados.

Palabras clave: Medicina herbaria; Cromatografia líquida de alta resolución (HPLC); Control de calidad.

\section{Introdução}

A Medicina Tradicional Chinesa (MTC) é uma ciência milenar que foi se modificando e agregando conhecimentos durante toda a sua história pelos seus praticantes (Lin, 2013). Esta prática baseia-se na divisão do universo entre dois princípios: yin e yang, que são subdivididos em fases de regulação, e são estas fases que mantém o organismo em equilíbrio (Melo, 2014). O homem, sendo parte da natureza, também precisa manter seu equilíbrio, e quando há um desequilíbrio, o organismo é afetado das mais diversas formas, como o aparecimento de doenças físicas e emocionais (Martini; Cardoso; Santos, 2009).

A utilização de ervas e partes de plantas para tratamento de enfermidades que são causadas por esse desequilíbrio é um dos modelos mais conhecidos de utilização da medicina chinesa e está presente em sua história há mais de 4.000 anos (Vieira; Motta \& Pereira, 2018). Segundo a MTC as plantas possuem sua própria energia, podendo auxiliar no equilíbrio energético do indivíduo, ou atuar em sinergismo com a energia de outras matérias (Bonaparte; Nardi, 2014).

$\mathrm{Na}$ crença que envolve os princípios da MTC, a energia que faz parte de cada um dos seres vivos possui algumas classificações, essas podem ser diferenciadas como tipos de temperatura, de sabores ou de direções, é o que demonstra em qual local haverá o efeito desejado e o reestabelecimento do equilíbrio (Bonaparte; Nardi, 2014).

Atualmente existem diversas formas farmacêuticas para veicular ativos farmacológicos, como as soluções. Essas apresentam como vantagem a facilidade de administração e maior rapidez na absorção através do trato gastrointestinal (Almeida et al, 2018). Na etapa de desenvolvimento do novo medicamento, a formulação deve passar por testes de controle de 
qualidade, que visam verificar se as características do novo produto estão sendo mantidas durante um determinado período, em condições controladas de temperatura e umidade (Costa; Lopes, 2016).

Dessa maneira, o presente trabalho teve como objetivo a realização de um estudo de estabilidade acelerado, por meio de testes de controle de qualidade físico-químicos e microbiológicos, de uma solução preparada com extrato de seis plantas da MTC: Rehmannia radix preparata, Fructus corni officinalis, Radix dioscorea oppositae, Sclerotiumporia cocos, Cortex moutan radicis e Rhizoma alismatis orientalis.

\section{Metodologia}

Esta é uma pesquisa experimental do tipo quantitativa (Koche, 2011). Neste trabalho foi realizado o estudo de estabilidade acelerado (6 meses) de solução fitoterápica contendo extrato de seis plantas da MTC. Foi utilizada câmara climática com controle de temperatura e umidade, à $40{ }^{\circ} \mathrm{C}$ e $25 \%$ UR. Em períodos pré-determinados, a formulação teste e o controle (solução sem extrato) foram avaliadas com relação aos parâmetros físico-químicos (características organolépticas, pH, densidade e viscosidade), microbiológicos e o perfil cromatográfico em UPLC.

\section{Determinação das características organolépticas}

Para as características organoléticas, foram analisados o aspecto, a cor e o odor, em comparação com o controle (solução sem extrato) (Pereira et al., 2004).

\section{Determinação do pH}

Foi realizada a determinação de $\mathrm{pH}$ em potenciômetro digital das soluções (teste e controle). A leitura foi realizada introduzindo o eletrodo do aparelho na amostra, procedendo três leituras sucessivas e obtendo a média (ANVISA, 2019).

\section{Determinação da densidade}

Foi realizada a determinação da densidade relativa das soluções (teste e controle) por meio do método do picnômetro, utilizando equipamento de vidro com capacidade de $50 \mathrm{~mL}$, previamente limpo e seco. A densidade foi calculada utilizando a equação (1) (ANVISA, 2019).

$$
d=(M 2-M 0) /(M 1-M 0)
$$

$$
\begin{aligned}
& \text { Sendo, } \\
& \text { M0: picnômetro vazio } \\
& \text { M1: picnômetro com água } \\
& \text { M2: picnômetro com a amostra }
\end{aligned}
$$

\section{Determinação da viscosidade}

A determinação da viscosidade foi realizada utilizando viscosímetro rotativo modelo ViscoSTAr-R (Fungilab, Espanha), sensor de acoplamento com spindle R2 a 100 rotações/min. As amostras foram adicionadas no recipiente coletor do aparelho e realizou-se a imersão do spindle nas amostras procedendo-se a leitura da viscosidade, e em seguida foi calculada a média (ANVISA, 2019). 


\section{Análise do perfil cromatográfico}

As análises foram realizadas em cromatógrafo líquido UltiMate 3000 (Thermo Scientific), equipado com detector de arranjo de diodos e software controlador (Chromeleon®). Foi utilizada uma coluna Phenomenex® Onix Monolithic C18 (100mm x 4.6mm Å, $2 \mu \mathrm{m})$ e Phenomenex coluna de guarda. Foram utilizadas as fases móveis A: água + 0,1\% ácido fosfórico e B: acetonitrila + 0,1\% ácido fosfórico, com a seguinte eluição gradiente: 0 min: $90 \%$ de A; 3 min: $80 \%$ de A; 10 min: $60 \%$ de A; 30 min: $80 \%$ de A. Como marcador químico, foi utilizada a substância padrão acteosídeo, presente na Rehmania preparata, solubilizada em metanol na concentração de $1 \mathrm{mg} / \mathrm{mL}$. A temperatura da coluna foi mantida a $25^{\circ} \mathrm{C}$ e a vazão de 0,5 mL/min. Como controle, foi utilizada uma solução contendo a mesma formulação, porém sem extrato. As soluções teste e controle não foram diluídas para a determinação do perfil cromatográfico.

\section{Análise microbiológica}

A determinação de microrganismos nas amostras (teste e controle) foi realizada empregando os meios TSA (para bactérias) e Sabouraud (para leveduras) enriquecidos com proteínas e carboidratos. O meio empregado para a diluição das amostras foi o MHB (Mueller Hinton Broth), e o método de semeadura foi Pour Plate (Tadoque, 2019). A análise foi realizada com todos os seus passos em duplicata.

As amostras foram diluídas 1:10 em tubos com tampa, totalizando $5 \mathrm{~mL}$. Após a homogeneização dos tubos, e a liquefação dos meios, esses foram adicionados em placa de Petri estéril, $1 \mathrm{~mL}$ da amostra (teste ou controle) e em seguida 15 mL do meio de cultura. Após a semeadura, a placa sofreu leve homogeneização. Por fim, após a solidificação desses meios, as placas foram incubadas a $32,0^{\circ} \mathrm{C}$ por 5 dias para verificação de crescimento microbiano.

\section{Resultados e Discussão}

\section{Determinação das características organolépticas}

A determinação das características organoléticas é realizada pelos sentidos de tato, paladar e olfato (Kindlein; Carvalho, 2015). Neste trabalho foram observadas as características organolépticas apresentadas na solução teste e comparadas com a solução controle, nos tempos zero, 3 e 6 meses, do estudo de estabilidade acelerada.

Os resultados encontrados podem ser observados na Tabela 1.

Tabela 1: Resultados das características organolépticas da solução teste.

\begin{tabular}{cccc}
\hline $\begin{array}{c}\text { Tempo } \\
(\text { mês })\end{array}$ & Odor & Cor & Aspecto \\
\hline 0 & Sem alteração & Vermelho escuro & Sem alteração \\
3 & Sem alteração & Vermelho escuro & Sem alteração \\
6 & Sem alteração & Vermelho escuro & Sem alteração \\
\hline
\end{tabular}

Fonte: Autores.

Durante o estudo de estabilidade acelerada não foi observado alteração nos parâmetros odor, cor e aspecto.

Em estudo semelhante, Oliveira (2017) avaliou as características organolépticas de soluções de guaco, e as amostras analisadas também mantiveram suas características nos tempos analisados. 
Determinação do $\mathrm{pH}$

A determinação do $\mathrm{pH}$ pode demonstrar se há problemas de estabilidade da amostra, pois possíveis reações entre os componentes podem levar a degradação de substâncias ativas e consequente alteração do pH da solução (Ferreira; Souza, 2011). Os resultados obtidos para esta análise, para a solução teste e controle, estão demonstrados na Tabela 2.

Tabela 2: Resultados do $\mathrm{pH}$ das soluções teste e controle.

\begin{tabular}{ccc}
\hline & $\mathbf{p H}$ & \\
\hline $\begin{array}{c}\text { Tempo } \\
(\text { mês })\end{array}$ & $\begin{array}{c}\text { Solução controle } \\
\text { Média } \pm \mathbf{D P *}\end{array}$ & $\begin{array}{c}\text { Solução teste } \\
\text { Média } \pm \mathbf{D P *}\end{array}$ \\
\hline 0 & $6,68 \pm 0,27$ & $4,62 \pm 0,23$ \\
1 & $6,17 \pm 0,23$ & $4,62 \pm 0,23$ \\
2 & $5,90 \pm 0,20$ & $4,27 \pm 0,01$ \\
3 & $5,84 \pm 0,02$ & $5,23 \pm 0,34$ \\
4 & $6,45 \pm 0,27$ & $4,54 \pm 0,34$ \\
5 & $6,00 \pm 0,29$ & $4,29 \pm 0,03$ \\
6 & $4,80 \pm 0,18$ & $3,62 \pm 0,18$ \\
\hline
\end{tabular}

*DP: Desvio Padrão. Fonte: Autores.

Pode-se observar valores de $\mathrm{pH}$ mais baixos para a solução contendo extratos vegetais. Em ambas soluções (teste e controle) verificou-se uma redução do pH no último tempo analisado (6 meses), indicando possível alteração dos constituintes nas formulações.

Silva e Tescarollo (2020), em estudo de avaliação da qualidade de soluções orais de paracetamol, demonstram que os valores de pH variaram entre 3,8 a 6,5. No estudo de Santos, Aragão e Furlan (2009), que avaliaram soluções de paracetamol oral, também houve semelhança nos resultados encontrados nas soluções, com tendência a pH ácido.

\section{Determinação da densidade}

A densidade é uma propriedade avaliada a partir da massa e do volume de uma amostra (Sampaio; Silva, 2007). Quando a densidade é avaliada em temperaturas diferentes, as moléculas da amostra estarão dispersas de formas diferentes, isso influencia diretamente no valor da densidade. Assim, tal propriedade é avaliada em temperatura padrão (20 ${ }^{\circ} \mathrm{C}$ ) (ANVISA, 2019). Os valores de densidade apresentados pelas soluções teste e controle podem ser visualizados na Tabela 3. 
Tabela 3: Resultados da densidade das soluções teste e controle.

\begin{tabular}{ccc}
\hline & \multicolumn{2}{c}{ Densidade $(\mathbf{g} / \mathbf{m L})$} \\
\hline $\begin{array}{c}\text { Tempo } \\
(\mathrm{mês})\end{array}$ & $\begin{array}{c}\text { Solução controle } \\
\text { Média } \pm \mathbf{D P *}\end{array}$ & $\begin{array}{c}\text { Solução teste } \\
\text { Média } \pm \mathbf{D P *}\end{array}$ \\
\hline 0 & $1,11321 \pm 0,00192$ & $1,11253 \pm 0,00141$ \\
1 & $1,11349 \pm 0,00045$ & $1,11253 \pm 0,00141$ \\
2 & $1,11325 \pm 0,00055$ & $1,11409 \pm 0,00054$ \\
3 & $1,11293 \pm 0,00045$ & $1,11324 \pm 0,00950$ \\
4 & $1,11213 \pm 0,00113$ & $1,11381 \pm 0,00113$ \\
5 & $1,11638 \pm 0,00574$ & $1,11758 \pm 0,00611$ \\
6 & $1,11327 \pm 0,00054$ & $1,11490 \pm 0,00028$ \\
\hline
\end{tabular}

*DP: Desvio Padrão. Fonte: Autores.

Brandão (2001) avaliou soluções aquosas, e os valores de densidade variaram entre 1,2 a 1,32 g/mL, na temperatura de $20^{\circ} \mathrm{C}$.

Delgado et al. (2012) avaliaram a densidade de uma solução oxidante contendo persulfato de sódio, obtendo valor médio de 1,02 g/mL. Em outro estudo, Mello (2004) avaliou preparações líquidas orais de maleato de enalapril na terapia hospitalar da insuficiência cardíaca congestiva, obtendo valor médio de densidade de 1,26 g/mL.

Todos os valores obtidos nos estudos citados vão ao encontro dos resultados obtidos no presente estudo.

\section{Determinação da viscosidade}

O nível de viscosidade é essencial para a formação final da textura do produto (Alves et al., 2014). Os valores obtidos na análise da viscosidade para as soluções teste e controle, em todos os tempos analisados, podem ser observados na tabela 4.

Tabela 4: Resultados da viscosidade das soluções teste e controle.

\begin{tabular}{ccc}
\hline & Viscosidade $(\mathbf{m P a} . \mathbf{)})$ & \\
\hline $\begin{array}{c}\text { Tempo } \\
(\mathrm{mês})\end{array}$ & $\begin{array}{c}\text { Solução controle } \\
\text { Média } \pm \mathbf{D P *}(\mathbf{T} \%)^{* *}\end{array}$ & $\begin{array}{c}\text { Solução teste } \\
\text { Média } \pm \mathbf{D P *}(\mathbf{T} \%)^{* *}\end{array}$ \\
\hline 0 & $15.1431 \pm 3,5$ & $18.8502 \pm 4,4$ \\
1 & $14.6730 \pm 3,4$ & $18.8502 \pm 4,4$ \\
2 & $14.6686 \pm 3,4$ & $16.1044 \pm 3,8$ \\
3 & $15.9113 \pm 3,7$ & $17.4564 \pm 4,1$ \\
5 & $16.8142 \pm 3,9$ & $18.0527 \pm 4,2$ \\
6 & $15.3241 \pm 3,6$ & $17.0725 \pm 4,0$ \\
\hline
\end{tabular}

*DP: Desvio Padrão.

$* * \mathrm{~T}(\%)$ : Torque.

Fonte: Autores. 
Lubi, Sato e Gaensly (2003) avaliaram o desenvolvimento de uma forma farmacêutica líquida de uso oral com extrato de guaco, e obtiveram valores de viscosidade próximos a $40 \mathrm{mPa}$.s. Em outro estudo, Lubi (2002), foi avaliado o desenvolvimento de forma farmacêutica líquida a base de guaco isenta de açúcar, apresentando resultados de 3.500 a 5.000 mPa.s.

Os valores encontrados no presente trabalho são diferentes dos valores obtidos nos artigos citados. Esta diferença pode estar relacionada com a composição das soluções, uso de agentes doadores de viscosidade, entre outros.

\section{Perfil cromatográfico}

A cromatografia é uma técnica onde os compostos são separados de acordo com sua relação com a fase estacionária e a fase móvel. Cada substância é detectada pelo detector acoplado ao equipamento e transformada em picos visíveis no cromatograma, pelo software utilizado (Paulino, 2020).

A avaliação por UHPLC foi realizada no início dos experimentos, após um mês e após dois meses, correspondendo aos tempos 0 (zero), 1 (um) e 2 (dois), respectivamente. Nos meses subsequentes ao tempo 2, observou-se um processo de alteração das substâncias e, desta forma, a análise cromatográfica não foi realizada durante todo o período do estudo de estabilidade acelerada.

Os cromatogramas referentes a solução padrão de acteosídeo, solução controle (solução sem extratos vegetais) e solução teste (contendo extratos vegetais) estão representados na Figura 1. 


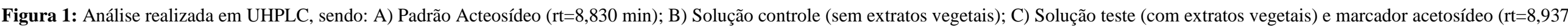
$\min ) . \mathrm{rt}=$ tempo de retenção

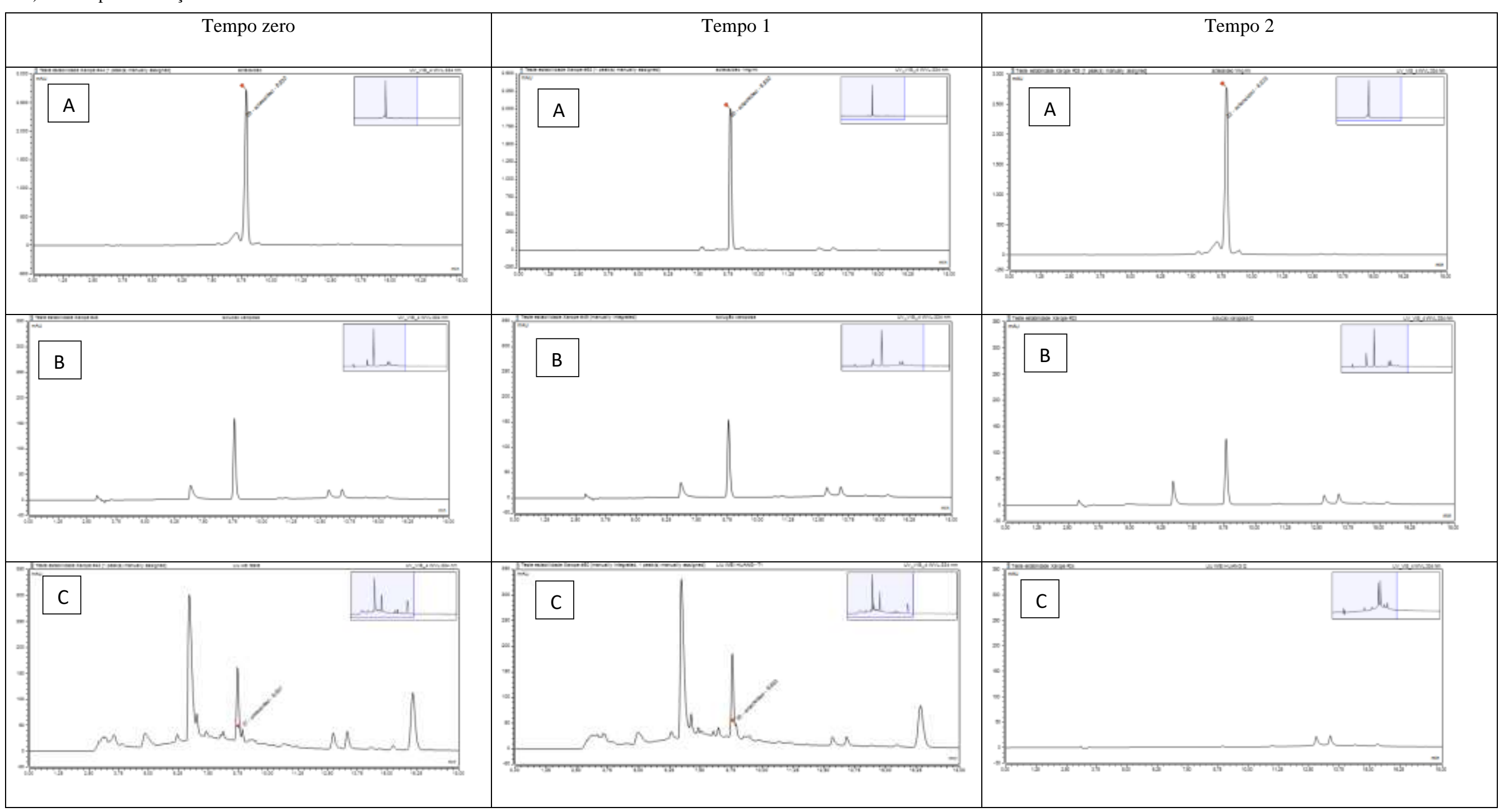




\section{Análise Microbiológica}

A análise microbiológica existe pela necessidade de garantir a segurança e eficácia dos produtos. A existência de microrganismos não intencionais na amostra, age diretamente na estabilidade da solução, alterando características químicas e físicas do produto (Nascimento et al., 2005; Yamamoto et al., 2004).

Para avaliar se a conservação e estabilidade das soluções (teste e controle) foram eficientes nas condições de temperatura e umidade em que foram expostas durante o estudo, a análise microbiológica foi realizada nos tempos 0,3 e 6 meses, e os resultados podem ser observados nas tabelas 5 e 6.

Tabela 5. Resultados da análise microbiológica das amostras em TSA (trypticase soy agar)

\begin{tabular}{|c|c|c|}
\hline \multicolumn{3}{|c|}{ TSA } \\
\hline $\begin{array}{c}\text { Tempo } \\
\text { (mês) }\end{array}$ & Solução sem ativos vegetais & Solução com ativos vegetais \\
\hline 0 & NEGATIVO & NEGATIVO \\
\hline 3 & NEGATIVO & NEGATIVO \\
\hline 6 & NEGATIVO & NEGATIVO \\
\hline
\end{tabular}

Fonte: Autores.

Tabela 6. Resultados da análise microbiológica das amostras em meio Sabouroad.

\begin{tabular}{ccl}
\hline \multicolumn{1}{c}{ Sabouraud } & \\
\hline Tempo & Solução sem ativos vegetais & Solução com ativos vegetais \\
\hline 0 & NEGATIVO & NEGATIVO \\
3 & NEGATIVO & NEGATIVO \\
6 & NEGATIVO & NEGATIVO \\
\hline
\end{tabular}

Fonte: Autores.

O limite máximo de unidades formadoras de colônia (UFC) permitidos tanto para bactérias quanto para fungos, são de $10^{2}$ UFC/mL para soluções (ANVISA, 2019). Nas amostras analisadas houve ausência de crescimento de bactérias e fungos durante as análises.

Estudo realizado por Santos (2018) que avaliou a qualidade microbiológica de solução oral de dipirona, apresentou crescimento inferior ao limite estabelecido pela legislação para a maioria das amostras, com cinco amostras apresentando em média $10^{1} \mathrm{UFC} / \mathrm{mL}$ para bactérias e para fungos, e apenas uma das oito amostras apresentou valor fora do limite estabelecido.

Em outro estudo semelhante, Lima (2010) avaliou a estabilidade microbiológica de extrato aquoso de própolis e obteve resultados variáveis, sendo tanto negativos quanto extremamente positivos; essa situação pode ter ocorrido por falhas preventivas e de controle de qualidade durante o processo de fabricação ou pela preparação conter substâncias que favoreçam o desenvolvimento de bactérias e/ou fungos (Yamamoto, 2000).

Desta forma, conclui-se que a análise realizada no presente trabalho apresentou resultados satisfatórios, já que as condições de temperatura e umidade não afetaram a segurança microbiológica das soluções. 
A degradação ativa de um composto pode ocorrer por diversos fatores, os quais são divididos em extrínsecos (temperatura, umidade, luz) e intrínsecos (oxidação e hidrólise) (Mirco; Rocha, 2014). Supõe-se que a degradação que aconteceu na formulação analisada foi uma degradação intrínseca e que tenha ocorrido por degradação dos ativos vegetais.

\section{Conclusão}

Os resultados observados nas avaliações físico-químicas realizadas durante o período de estudo (6 meses) demonstraram estabilidade adequada nos parâmetros organolépticos, $\mathrm{pH}$ e densidade. A viscosidade observada foi diferente de outros trabalhos, mas é aceitável para soluções e manteve-se estável durante os tempos analisados. Já na avaliação por cromatografia, observou-se indícios de degradação de substâncias após o tempo 1 mês, não apresentando estabilidade diante das condições de temperatura e umidade utilizadas durante o estudo. Na avaliação da qualidade microbiológica, não foi constatado o crescimento de fungos ou bactérias nos tempos analisados.

Para trabalhos futuros, seria interessante testar formulações com excipientes que melhorem a estabilidade das substâncias ativas, como sistemas nanoestruturados contendo os extratos.

\section{Referências}

Agência Nacional de Vigilância Sanitária (ANVISA). (2019). Farmacopeia Brasileira. (6a ed.),

Agência Nacional de Vigilância Sanitária (ANVISA). (2004). Guia de Estabilidade de Produtos Cosméticos. v.1.

Almeida, J., Pasqualotto, J., Lopes, A. C., Oliveira, C. F., Betim, F. C. M., Martinez, A. L. \& Bobek, V. B. (2018). Xarope Broncodilatador Adulto - Estudo físico-químico. Biblioteca digital de periódicos.

Bonaparte, L. F. \& Nardi, C. M (2014). Fitoterapia Chinesa - Breve Histórico de Uso Complementar a Tratamentos de Saúde na Medicina Tradicional Chinesa: Revisão Bibliográfica. <https://docplayer.com.br/12710662-Fitoterapia-chinesa-breve-historico-de-uso-complementar-a-tratamentos-de-saude-namedicina-tradicional-chinesa-revisao-bibliografica.html >. .

Brandão, A. (2001). Controle da Qualidade e Controle da Produção de Medicamentos. Ensaios para Laboratório de Controle da Qualidade e Controle da Produção de Medicamentos. <http://www.boaspraticasfarmaceuticas.com.br/includes/Controle_da_qualidade.doc>.

Costa, T. M. F. \& Lopes, J. C (2016). Controle de Qualidade de Soluções Orais de Dipirona sódica 500mg/mL Referência, Genérico e Similar Comercializadas em Gurupi-TO. Revista Amazônia Science \& Health. Gurupi.

Delgado, A. V. B., Cardoso, D. \& Oliveira, E. (2012). Observação de Efeitos de Densidade na Solução Oxidante de Persulfato de Sódio em Aquífero Integranular e Aquífero fraturado contaminado por Etenos Clorados. XVII Congresso Brasileiro de Águas Subterrâneas e XVIII Encontro Nacional de Perfuradores de Poços. Revista águas subterrâneas, Bonito.

Ferreira, A. O. \& Souza, G. F. (2011). Preparações Orais Líquidas. Pharmabooks, (2a ed.).

Kindlein, L. \& Carvalho, H. R. (2015). Características Organolépticas Responsáveis pela Rejeição do Pescado Fresco do Ponto de Vista do Consumidor. Porto Alegre. < https://lume.ufrgs.br/bitstream/handle/10183/166779/Resumo_29454.pdf?sequence=1\&isAllowed=y>.

Koche, J. C. (2011). Fundamentos de metodologia científica. Petrópolis: Revista Vozes. <https://repositorio.ufsm.br/bitst ream/handle/1/15824/Li c_Computacao_Metodologia-Pesquisa-Cientifica.pdf?sequence=1>.

Lin, C. A. (2013). Da Medicina Tradicional Chinesa à Prática de Acupuntura Médica Baseada em Evidência. Universidade de São Paulo, Faculdade de Medicina. Revista de medicina. São Paulo.

Lubi, N. C. (2002). Desenvolvimento de Forma Farmacêutica Líquida de Uso Oral, Isenta de Açúcar com Extrato Fluido de Guaco (Mikania glomerata Sprendel, Asteracceae), para Afecções do Aparelho Respiratório. <https://www.acervodigital.ufpr.br/bitstream/handle/1884/27633/D\%20$\% 20$ LUBI\%2c\%20NEIVA\%20CRISTINA.pdf?sequence=1\&isAllowed=y $>$.

Lubi, N. C., Sato, M. E. O. \& Gaensly, F. (2003). Desenvolvimento de Forma Farmacêutica Líquida de uso oral, Isenta de Substâncias Glicogênicas, com Extrato Fluido de Mikania glomerata Sprengel - Asteraceae (guaco). Revista Brasileira de Farmacognosia, v. 13. $<$ https://www.scielo.br/j/rbfar/a/4pCBNfNwLNQ6zKJnyH6Jr4g/?format=pdf\&lang=pt>.

Martini, L., Cardoso, M. \& Santos, M.C. (2009). Medicina Tradicional Chinesa no Tratamento da Obesidade. <http://siaibib01.univali.br/pdf/laraine\%20martini\%20e\%20maisa\%20cardoso.pdf>

Melo, S.R.F. (2014). Determinação In Vitro da Atividade Antibacteriana da Artemísia vulgaris, Coptis chinensis e Scutellaria barbata: Comparação entre Infusão, Decocção e Óleo Essencial. <https://repositorio-aberto.up.pt/bitstream/10216/77795/2/33840.pdf>. 
Mirco, J. \& Rocha, M. S. (2014). Estudo de Estabilidade de Medicamentos. Revista acadêmica Oswaldo Cruz.

Nascimento, V.T., Lacerda, E. U., Melo, J. G., Lima, C. S. A., Amorim, E. L. C. \& Albuquerque, U.P. (2005). Controle de Qualidade de Produtos à Base de Plantas Medicinais Comercializadas na Cidade do Recipe-PE: erva-doce (Pimpinella anisum L.), quebra-pedra (Phyllanthus spp.), espinheira santa (Maytenus ilicifolia Mart.) e camomila (Matricaria recutita L.). Revista Acta farmacêutica bonaerense

Oliveira, L. B. B. (2017). Avaliação Microbiológica de Xaropes Fitoterápicos Contendo Mikania glomerata S., Comercializados no Município de Santo Antônio de Jesus - BA. Repositório institucional faculdade Maria Milza. Santo Antônio de Jesus.

Paulino, R. R. (2020). Cromatografia Líquida, Avanços e Inovações. Universidade de Coimbra. Coimbra. <https://estudogeral.uc.pt/bitstream/ 10316/93060/1/cromatografia\%20FINAL.pdf>

Pereira, A. V., Farago, P. V., Pereira, F. P. M., Brito, F. S. \& Scaranello, V. F. L. (2004). Análise de soluções magistrais de Curcuma zedoaria (BERGIUS) ROSCOE. UEPG Ci. Biol. Saúde. Revista publicatio UEPG.

Sampaio, J. A. \& Silva, F. A. N. (2007). Determinação das Densidades de Sólidos e de Polpa. CETEM/MTC. <http://mineralis.cetem.gov.b r/bitstream /cetem/1019/1/Cap\%202\%20Densidade\%20Final.pdf>.

Santos, G. N., Aragão, C. C. V. \& Furlan, C. M. (2009). Estudo da Estabilidade Acelerada de Solução Oral de Paracetamol 200mg/mL, Distribuída no Sistema Único de Saúde - SUS. Revista Brasileira de Ciências de Saúde. São Caetano do SUL.

Silva, E. M. \& Tescarollo, I. L. (2019). Avaliação da Qualidade de Soluções Orais de Paracetamol. Revista Ensaios Pioneiros.

Tardoque, D. W. A. (2019) Análise Microbiológica da Água do Instituto de Saúde de Nova Friburgo da Universidade Federal Fluminense. Instituto de saúde de Nova Friburgo. <https://app.uff.br/riuff/handle/1/12592>.

Vieira, A.J., Motta, R.N.C. \& Pereira, L.P. (2018). Introdução da Fitoterapia Chinesa no Sistema Único De Saúde (SUS). Revista Saúde em Foco, (10a ed.).

Yamamoto, C. H., Pinto, T. J. A., Meurer, V. M., Carvalho, A. M. \& Rezende, P. (2004). Controle de Qualidade Microbiológico de Produtos Farmacêuticos, Cosméticos e Fitoterápicos Produzidos na Zona da Mata, MG. Anais do $2^{\circ}$ Congresso Brasileiro de Extensão Universitária.

Yamamoto C. H., Alves, M. S., Pinto T. J. A. \& Oliveira, I. F. (2000) Contaminação Microbiana em Matérias-Primas de Origem Natural Empregadas em Farmácia de Manipulação. Farmácia e Química. Universidade Federal de Juiz de Fora, São Paulo. 